
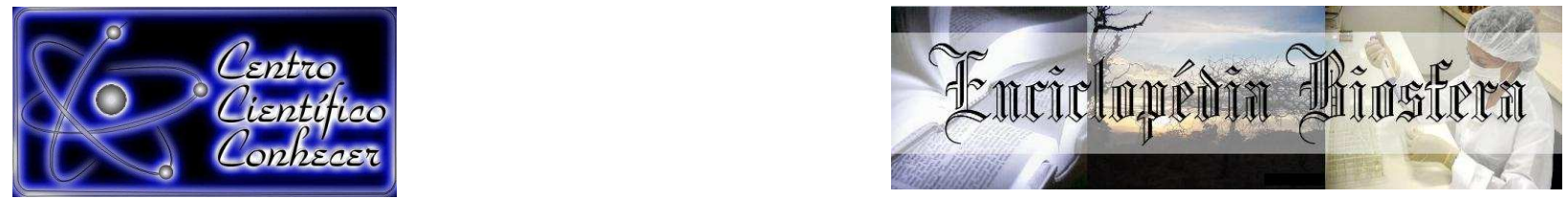

\title{
PERFIL DOS COMERCIANTES DE PLANTAS MEDICINAIS NO MUNICÍPIO DE URUARÁ, PARÁ, BRASIL
}

Reinaldo Lucas Cajaiba ${ }^{1}$, Anderson Franco Gomes ${ }^{2}$, Maria do Carmo Santos ${ }^{2}$, Rosilane Rodrigues de Medeiros ${ }^{2}$, Wully Barreto da Silva ${ }^{3}$

${ }^{1}$ Biólogo, Doutorando em Ambiente e Desenvolvimento, Univates, RS (reinaldocajaiba@hotmail.com)

${ }^{2}$ Tecnólogo em Gestão Ambiental, Faeco

${ }^{3}$ Universidade Federal do Pará-UFPA

Recebido em: 03/10/2016 - Aprovado em: 21/11/2016 - Publicado em: 05/12/2016 DOI: 10.18677/EnciBio_2016B_136

\begin{abstract}
RESUMO
Objetivou-se com este estudo analisar o perfil socioeconômico dos comerciantes de produtos e plantas medicinais do município de Uruará, Pará. Para tanto, foram realizadas entrevistas semi estruturadas com todos os comerciantes existentes na zona urbana do município. Como resultado, verificou-se que a maioria (56\%) trabalham em suas residências, seguido por mercado municipal (22\%) e em pontos comerciais distribuídos pela cidade (22\%). A faixa etária dos comerciantes variou de 37 a 72 anos. Observou-se que o percentual de pessoas com ensino fundamental incompleto manteve-se acima da metade da amostra (56\%), e fundamental completo representando 22\%; já o ensino médio incompleto representou $11 \%$ dos entrevistados, valor igual para o ensino superior. A comercialização de plantas e produtos medicinais é a principal fonte de renda dos entrevistados (67\%), e que os mesmos têm o apoio de familiares ou amigos para o desenvolvimento das atividades. Os principais fatores que levaram os comerciantes a desenvolverem tais atividades foram a tradição familiar $(44 \%)$ e necessidade de elevar a renda (34\%). Em $67 \%$ dos casos, a família, representado pela figura dos pais, tios e avós são os principais responsáveis pela transmissão dos conhecimentos, sendo que as informações são repassadas aos mais novos através da transmissão oral. Do ponto de vista cultural, o comércio de produtos e plantas medicinais é um sistema dinâmico, onde existe um sistema médico compartilhado pela grande maioria dos vendedores.
\end{abstract}

PALAVRAS-CHAVE: Amazônica; Conhecimento popular; Plantas medicinais.

\section{PROFILE OF MERCHANTS OF MEDICINAL PLANTS OF THE MUNICIPALITY OF URUARÁ, PARÁ, BRAZIL}

\begin{abstract}
The present study had as objective to analyze the socioeconomic profile of merchants of products and medicinal plants of the urban area of the municipality of Uruará, Pará. For this purpose, semi-structured interviews were conducted with all existing merchants in the urban area of the municipality. As a result, it was found that ENCICLOPÉDIA BIOSFERA, Centro Científico Conhecer - Goiânia, v.13 n.24; p.1473 2016
\end{abstract}


the majority (56\%) work from their homes, followed by municipal market $(22 \%)$ and stores in the city $(22 \%)$. The age-range of merchants ranged from 37 to 72 years, with an average of 51 years. It was observed that the percentage of people with incomplete elementary school remained above half the sample $(56 \%)$, the complete elementary school representing $22 \%$; the incomplete high school already represented $11 \%$ of respondents, equal value for higher education. The commercialization of plants and medicinal products is considered the main income source of the merchants of most respondents (67\%), and that they have the support of family or friends for the development of the activities. The main factors that led merchants to develop such activities were the family tradition (44\%) and need to increase the income (34\%). In $67 \%$ of cases, the family, represented by the figure of parents, uncles and grandparents are the main responsible for the transmission of knowledge and the information is transferred to the young people through oral transmission. From the cultural point of view, the trade in products and medicinal plants is a dynamic system, where there is a medical system shared by the vast majority of sellers.

KEYWORDS: Amazon; Popular knowledge; Medicinal plants.

\section{INTRODUÇÃO}

Desde os primórdios o ser humano recorre aos recursos naturais para a sobrevivência, o que inclui as plantas. Estas vêm sedo utilizadas para diversos fins, dentre estes destaca-se a utilização com finalidades medicinais no tratamento, na prevenção e na cura de doenças, não só em humanos como também em animais (CAJAIBA et al., 2016).

Tem crescido mundialmente nos últimos anos os interesses relacionados às plantas que são utilizadas como medicamentos, forma de utilização, finalidade e comercialização das mesmas. Isso tem resultado em um enorme crescimento da comercialização de plantas medicinas ou seus extratos (FREITAS et al., 2012). De acordo com a Organização Mundial de Saúde (OMS) $80 \%$ da população mundial recorrem às plantas para solucionar problemas de Saúde (ROCHA et al., 2013).

Os fatores que contribuíram para esse aumento foram vários, dentre estes esta o baixo custo, considerando que os valores dos medicamentos alopáticos são bem mais altos, a dificuldade que boa parte da população enfrenta para ter assistência médica, a precariedade nos sistemas públicos de saúde, ou até mesmo preferência por medicamentos naturais (BRASILEIRO et al., 2008; FREITAS et al., 2012).

Os mercados e feiras livres tradicionais são fundamentais por reunir, concentrar, manter e dissipar o conhecimento empírico sobre a diversidade de recursos tanto da fauna como da flora, sendo fontes indispensáveis para a resiliência e manutenção do conhecimento sobre as plantas medicinais (FREITAS et al., 2012; FERREIRA, 2014).

A utilização desses recursos terapêuticos pelas comunidades interioranas, e recentemente, nos grandes centros urbanos, se dá de forma contínua há décadas, seguindo relatos de especialistas tradicionais, que indicam a planta medicinal, preparam os remédios e orientam o usuário, baseando-se na experiência acumulada ao longo dos anos (CAJAIBA et al., 2016).

Diante do exposto, o presente trabalho objetivou analisar o perfil socioeconômico dos comerciantes de produtos e plantas medicinais da zona urbana do município de Uruará, Pará, envolvendo questões sobre idade, gênero, escolaridade, tempo de atuação no ramo, fatores que os levaram a entrar na 
atividade, renda média mensal obtida e forma de obtenção dos conhecimentos etnobotânicos.

\section{Local de estudo}

\section{MATERIAL E MÉTODOS}

A pesquisa foi realizada com os comerciantes de plantas e produtos medicinais na zona Urbana do município de Uruará-PA. O município de Uruará pertence à micro região de Altamira sendo localizado às margens da BR 230 , Rodovia Transamazônica, mais precisamente no km 180, a uma distância de 190 km da Cidade de Altamira. A sede Municipal apresenta as seguintes coordenadas

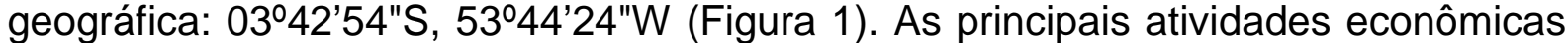
do município são: a agropecuária e a indústria madeireira (CAJAIBA et al., 2015).

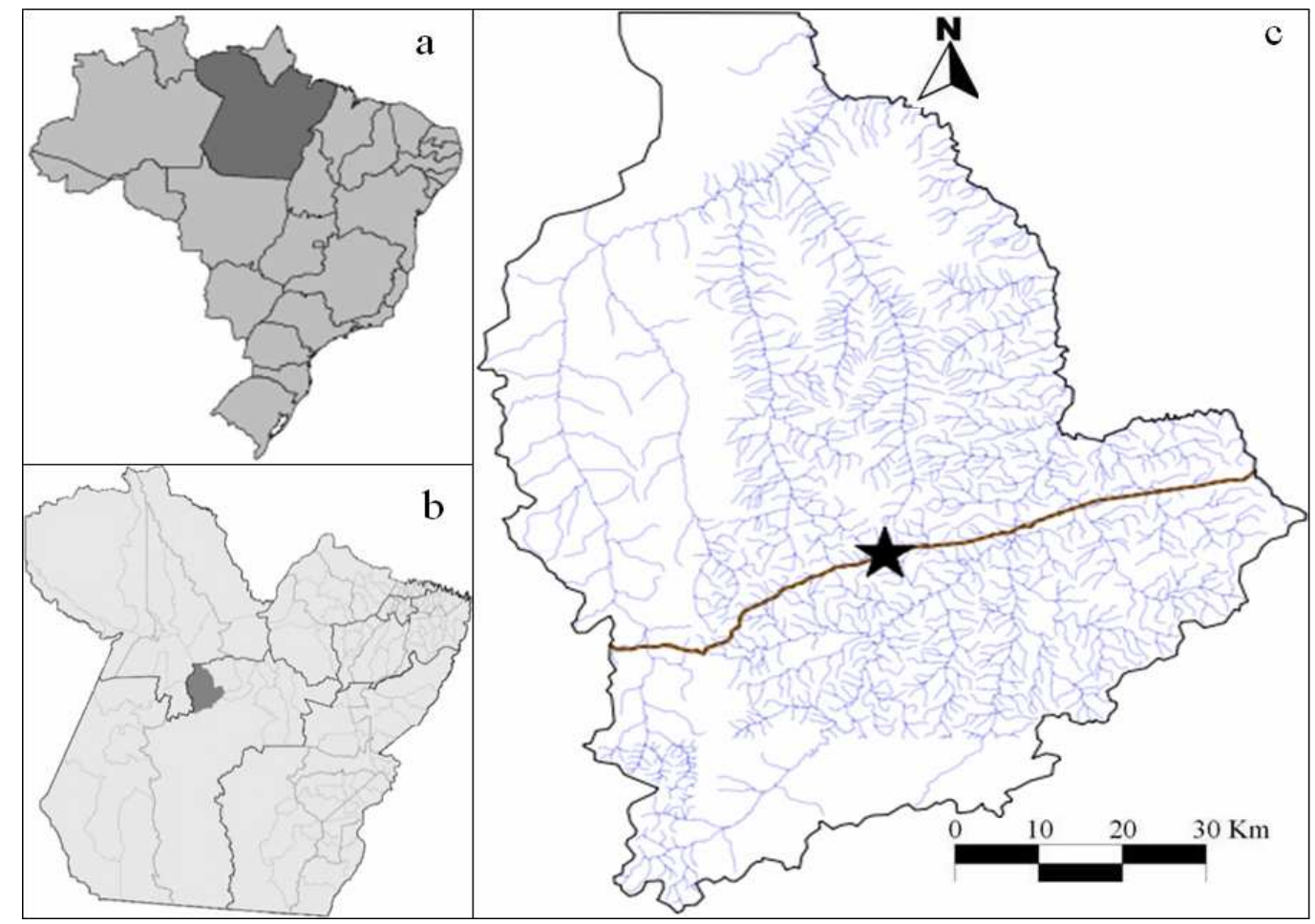

FIGURA 1. Localização do município estudado: a) Brasil, b) estado do Pará e c) município de Uruará com localização da área urbana (simbolizado pela estrela).

\section{Coleta de dados}

O trabalho de campo foi desenvolvido nos meses de fevereiro e março do ano de 2015, através de entrevistas semi estruturadas, contendo perguntas abertas e fechadas sobre o tema proposto e de observações diretas durante as entrevistas. Para a escolha dos entrevistados foi utilizada a técnica "Bola de Neve" (BAILEY, 1994), que implica em identificar os próximos a serem entrevistados a partir de indicações de anteriores. Tais indicações viabilizaram o reconhecimento de informantes-chave (BALDIN \& MUNHOZ, 2011), sendo estas pessoas da comunidade que são reconhecidas por deter um maior conhecimento e experiência sobre o tema pesquisado.

Quando houve a necessidade de confirmar a consistência e a veracidade de determinadas respostas, recorreu-se às entrevistas repetidas em situações 
sincrônicas e diacrônicas (MARQUES, 1991). Foram entrevistadas somente pessoas com idade igual ou superior a 18 anos e, prioritariamente os principais responsáveis pela comercialização dos produtos. Todos os entrevistados assinaram o Termo de Consentimento Livre e Esclarecido - TCLE.

\section{Análise dos dados}

Para realizar a análise dos dados, utilizou-se de abordagem qualitativa e quantitativa, conforme proposta por APPOLINÁRIO (2006). Os dados coletados foram tabulados através do programa Microsoft Excel®, para que, posteriormente, fosse realizada a análise descritiva do estudo.

\section{RESULTADOS E DISCUSSÃO}

Participaram da entrevista nove comerciantes de plantas e/ou produtos à base de plantas medicinais na zona urbana do município de Uruará, Pará. A comercialização desses produtos é praticada principalmente em suas residências (56\%), mas também são encontradas em pequenos estabelecimentos comerciais fixos no mercado municipal (22\%) e em pontos comerciais distribuídos em outros pontos da cidade (22\%). Dos entrevistados, $11 \%$ são homens e $89 \%$ mulheres. Este resultado se assemelha ao encontrado por FERREIRA (2014) em que a proporção de mulheres em relação aos homens que desenvolvem essa atividade é bem superior. Este fato pode estar associado aos aspectos culturais, já que, em diversos grupos sociais as mulheres costumam desenvolver atividades mais relacionadas ao âmbito doméstico, sobretudo em regiões do interior (FREITAS et al., 2012). A relevância das mulheres na detenção e dissipação do conhecimento popular já foi reconhecida em outros estudos etnobotânicos (CEOLIN et al., 2011; CAJAIBA et al., 2016).

A idade dos comerciantes variou entre 37 e 72 anos, com uma média de 51 anos $(\mathrm{dp} \pm 10,73)$. Esses dados são corroborados com vários outros estudos desenvolvidos em diferentes regiões do país (FREITAS et al., 2012; CAJAIBA et al. 2016). Resultado como este já era esperado, tendo em vista que pessoas mais idosas tendem a possuir um maior conhecimento relacionado às propriedades terapêuticas das plantas medicinais (ALVES et al., 2008). ARAUJO et al. (2009) consideram a falta de interesse das novas gerações uma ameaça ao repasse de informações valiosas no que diz respeito aos recursos vegetais medicinais da flora brasileira. Neste estudo foram encontrados dois perfis de vendedores, os com mais idade, que desde o início exerce essa profissão e os mais jovens que no início de carreira exerceram outra profissão e que por algum motivo recorreram a comercialização de ervas para a sobrevivência.

$O$ percentual de comerciantes com ensino fundamental incompleto manteve-se acima da metade da amostra (56\%), e fundamental completo representando $22 \%$; já o ensino médio incompleto representou $11 \%$ dos entrevistados, valor igual para o ensino superior. Apesar de a maioria dos entrevistados com ensino fundamental incompleto representar mais de $50 \%$, constatou-se que a escolaridade entre os raizeiros é relativamente alta quando comparada com a de outros estudos (ALVES et al., 2008; CAJAIBA et al. 2016).

Esse reduzido nível de estudo reforça a relevância da comercialização de plantas medicinais, pois, para muitos, essa é a única fonte de renda, e como em tal atividade se usa o conhecimento popular sobre as plantas que comercializa, não é 
necessário um alto grau de escolaridade para exercer essa profissão (CAJAIBA et al., 2016).

A renda mensal entre os comerciantes, decorrente dessa atividade, varia entre $R \$ 1.000,00$ a $R \$ 3.300,00$. No entanto, essa categoria nem sempre é fácil de ser avaliada, tendo em vista que se trata de uma economia informal (FREITAS et al., 2012). A renda mensal encontrada é bem superior quando se compara com outros estudos de outras regiões (DOURADO et al., 2005; ALVES et al., 2008).

A comercialização de plantas e produtos medicinais é a principal fonte de renda dos comerciantes, uma vez que $67 \%$ não desempenham outras atividades. Entretanto, 11\% comercializam outros produtos além dos de finalidade medicinais, dentre estas, verduras, frutas, legumes, temperos, dentre outros. Verificou-se que $22 \%$ dos comerciantes, além de comercializarem produtos e plantas medicinais, também desenvolvem o trabalho de "consulta médica", onde os interessados os procuram em seus "consultórios" e após o atendimento, recebem o "receituário" com os produtos a serem usados, que são comprados no mesmo estabelecimento onde foram consultados (Figura 2).

Esses resultados corroboram com os encontrados por DOURADO et al. (2005) que observaram que a maioria dos comerciantes trabalham exclusivamente com a venda de plantas medicinais, condimentares e aromáticas, evidenciando a importância socioeconômica dessa atividade (ALVES et al., 2008).

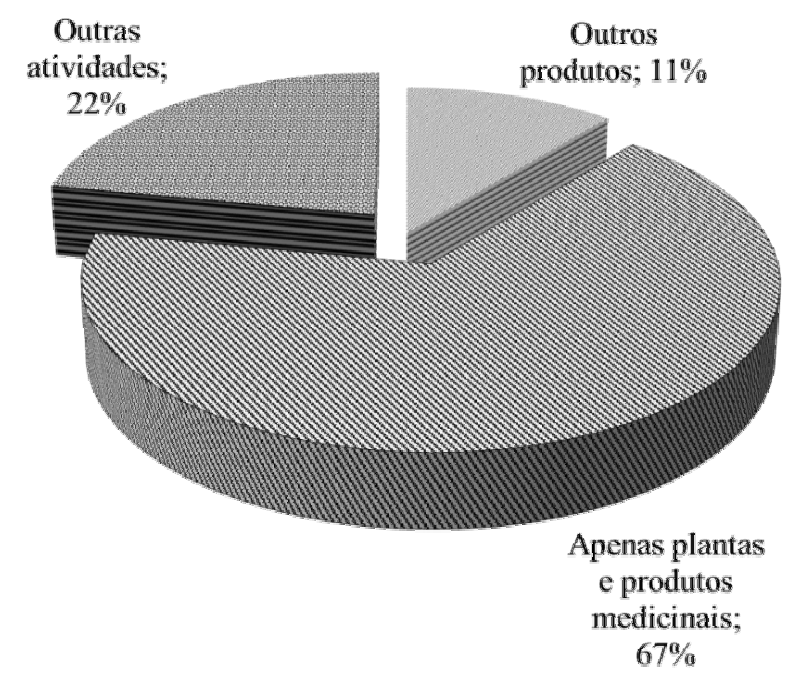

FIGURA 2. Principais fontes de renda dos comerciantes entrevistados.

Verificou-se também que todos os comerciantes entrevistados em Uruará têm o apoio de familiares ou amigos para o desenvolvimento das atividades, sendo assim distribuídas: filhos (40\%); esposa/ marido (40\%) e outros-nora, genro, afiliado $(20 \%)$ (Figura 3). 


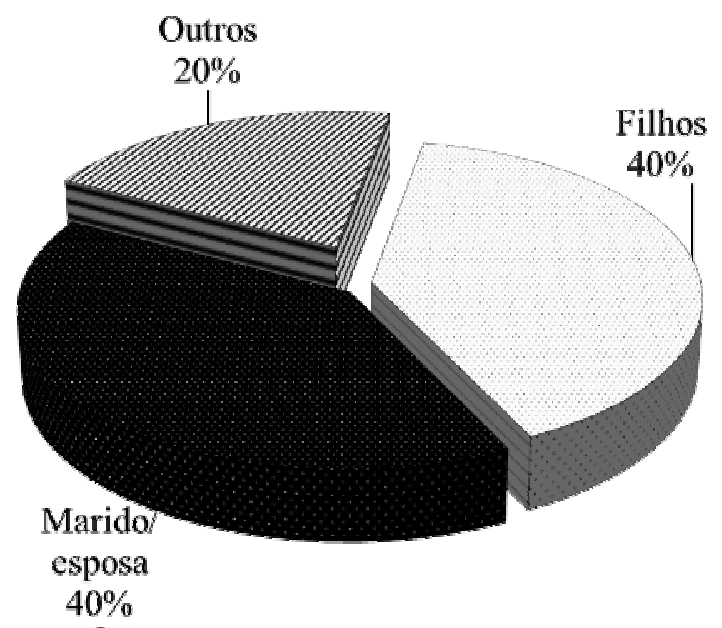

FIGURA 3. Grupo que ajudam a desempenhar a comercialização de produtos e plantas medicinais.

O tempo em que os entrevistados atuam nessa atividade de comercialização de plantas e produtos medicinais variou de sete a 30 anos, com média de 18 anos $(\mathrm{dp} \pm 7,17)$ na atividade, mostrando a relevância da atividade como geradora de emprego e renda. Resultados semelhantes foram encontrados por DOURADO et al. (2005), ALVES et al. (2008) e FREITAS et al. (2012).

Com relação à atividade desenvolvida anteriormente pelos vendedores, 56\% declararam que eram agricultores, $11 \%$ comerciantes e 33\% domésticas. Esses dados indicam que o comércio de produtos e plantas medicinais é exercido por pessoas que não estão inseridos ou não conseguem se inserir no mercado de trabalho formal. Essas informações são corroboradas quando os comerciantes foram questionados se trocavam de emprego, caso tivessem uma oferta ganhando o mesmo que ganham na atual atividade, $66 \%$ afirmaram que sim, alegando, inclusive a dificuldade de encontrar plantas medicinais para a produção dos produtos e demonstrando uma preocupação ao risco de extinção de algumas espécies.

Quando interrogados sobre quais as causas que os levaram a trabalhar nessa atividade, os entrevistados citaram a tradição familiar, valorizar as plantas/ produtos medicinais $(44 \%)$, a necessidade de complementar a renda (34\%), carência de vendedores desses produtos no município $(11 \%)$ e por fazer parte de projetos de extrativismo não madeireiro que conserva a floresta (11\%) (Figura 4). Assim, observa-se a importância da família no repasse de conhecimento e cultura para as próximas gerações (FREITAS et al., 2012). Resultados análogos foram encontrados por CAJAIBA et al. (2016) que consideraram como fatores fundamentais para a entrada na atividade o prosseguimento da tradição familiar, a não inserção no mercado de trabalho formal, evitar a ociosidade ao aposentar-se e por fatores diversos. 


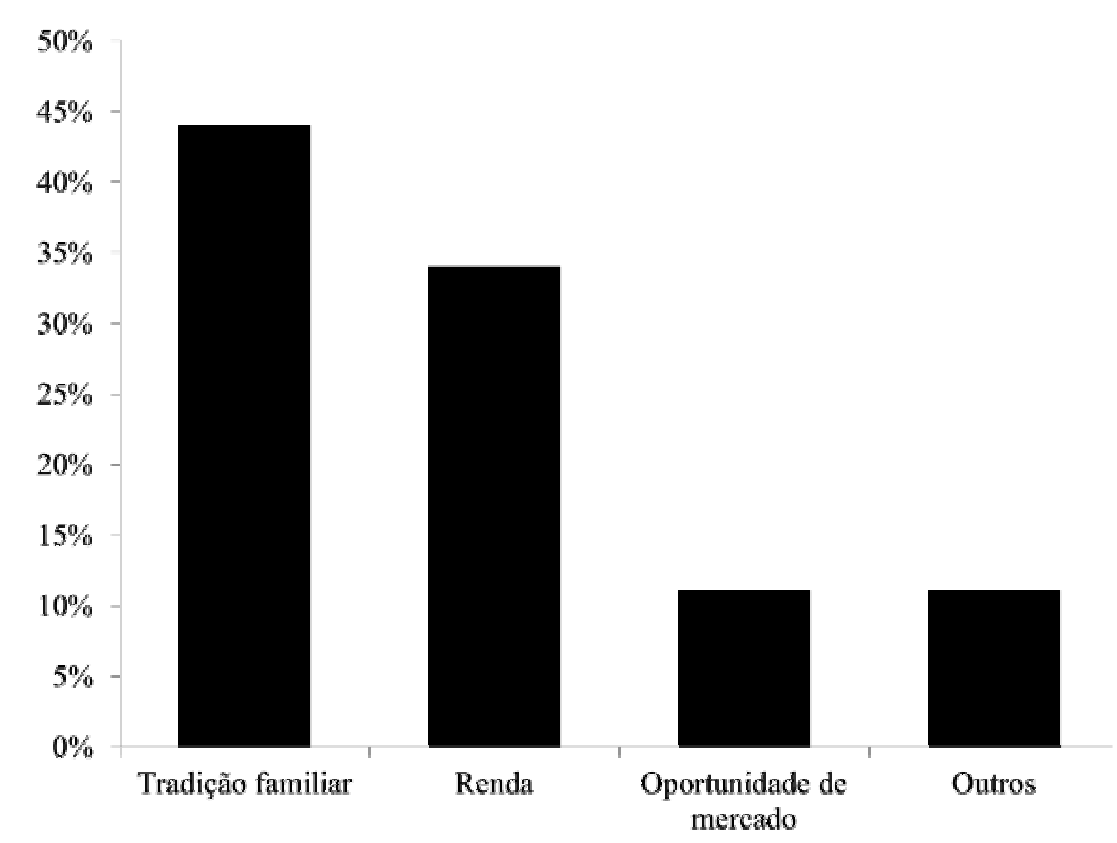

FIGURA 4. Motivos que levaram os entrevistados a comercializarem plantas e produtos medicinais.

A forma de obtenção do conhecimento sobre as plantas e produtos medicinais é através das mais variadas fontes (Figura 5). Sobre este aspecto, deve-se destacar a família $(67 \%)$, representados pela figura do pai, tio e avós, no repasse de conhecimentos a respeito das plantas medicinais, sugerindo que uma relação afetiva e de confiança facilita essa troca de saberes (FREITAS et al., 2012). Dos entrevistados, $22 \%$ informaram que além da família, obtém conhecimentos através de pessoas idosas, mais experientes no assunto, e 11\% afirmaram que buscam obter conhecimentos em livros, internet e revistas.

Segundo OLIVEIRA \& GONDIM (2013) grande parte do conhecimento sobre as plantas medicinais provém de uma vivência cotidiana de diferentes culturas humanas com a natureza, sendo que o principal modo de transmissão do conhecimento é o oral, através de tradição familiar, de gerações a gerações (AMOROZO, 2002). Todavia, as novas gerações no ambiente urbano buscam meios modernos de comunicação, incorporando programas de televisão, palestras, cursos, vídeos e internet (OLIVEIRA \& GONDIM, 2013). Porém, a continuidade pode ser ameaçada pela interferência de fatores externos à dinâmica social do grupo como, por exemplo: maior exposição das comunidades às pressões econômicas e culturais externas; maior facilidade de acesso aos serviços da medicina moderna; deslocamento das pessoas de seus ambientes naturais para regiões urbanas, o que leva à perda do conhecimento popular acumulado há várias gerações e, consequentemente, ao desaparecimento (HOEFFEL et al., 2011). 


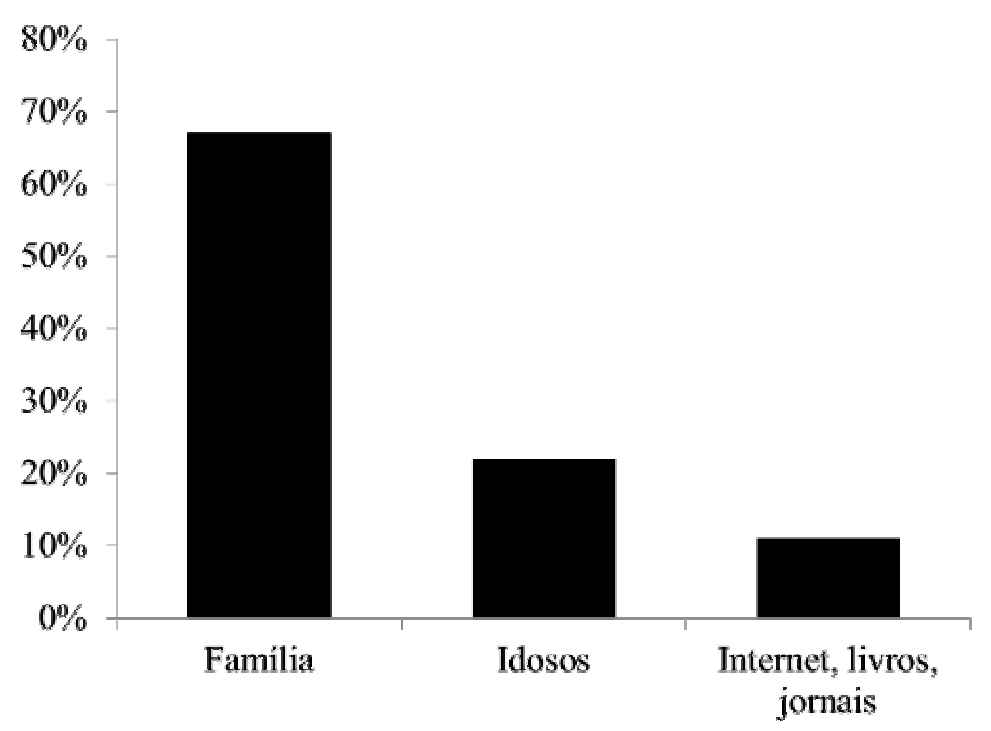

FIGURA 5. Forma de aprendizagem do uso de plantas medicinais.

Foi possível observar ainda, que o sistema cultural do comércio de plantas medicinais e seus produtos funcionam com base na partilha de saber. Quando um vendedor não possui o produto desejado pelo cliente, ele vai à busca dos demais vendedores que ofertam a mercadoria ou sugerem substitutos. Pode-se dizer, conforme afirmou PRESS (1978) para a medicina folk urbana, que o sistema cultural da feira é um sistema aberto, suscetível de receber diversas influências; está em constante transformação, como o próprio saber local, de uma sazonalidade bem clara de biorecursos e conhecimentos sobre o seu uso (ALMEIDA \& ALBUQUERQUE, 2002).

\section{CONCLUSÃO}

A pesquisa realizada junto aos comerciantes de produtos e plantas medicinais no município de Uruará permitiu concluir que a maioria dos comerciantes é do sexo feminino e que a maioria está numa faixa etária superior a 50 anos de idade, sendo que mais de $50 \%$ tem apenas o ensino fundamental incompleto. Conclui-se também, que a maioria dos entrevistados adquirem o conhecimento etnobotânico através de seus familiares, representados pela figura dos pais e dos avós e também dos idosos que desenvolvem essas atividades há mais tempo, sendo que as informações são repassadas aos mais novos através da transmissão oral.

\section{REFERÊNCIAS}

ALMEIDA, C.F.C.B.R.; ALBUQUERQUE, U.P. Uso e conservação de plantas e animais medicinais no estado de Pernambuco (nordeste do Brasil): Um estudo de caso. Interciência, v. 27, n. 6, p. 276-285, 2002.

ALVES, R.R.N.; SILVA, C.C.; ALVES, H.N. Aspectos socioeconômicos do comercio de plantas e animais medicinais em áreas metropolitanas do Norte e Nordeste do Brasil. Revista de Biologia e Ciências da Terra, v. 8, p. 181-189, 2008.

AMOROZO, M.C.M. Uso e diversidade de plantas medicinais em Santo Antônio de Leveger, MT, Brasil. Acta BotanicaBrasilica, v. 16, n. 2, p.189-203, 2002. 
APPOLINÁRIO, F. Metodologia da Ciência: Filosofia e Prática da Pesquisa. São Paulo: Pioneira Thomson Leraning, 2006.

ARAUJO, A.C., SILVA, J.P., CUNHA, J.L.X.L.; ARAUJO, J.L.O. Caracterização socio-econômico-cultural de raizeiros e procedimentos pós-colheita de plantas medicinais comercializadas em Maceió, AL. Revista Brasileira de Plantas Medicinais, v. 11, 81-91, 2009.

BAILEY, K. 1994. Methods of social research. New York: The Free Press. 591 p.

BALDIN, N.; MUNHOZ, E.M.B. Educação Ambiental comunitária: Uma experiência com a técnica de pesquisa snowball (bola de neve). Revista eletrônica Mestrado em Educação Ambiental, v. 27, p. 46-60, 2011.

BRASILEIRO, B.G.; PIZZIOLO, V.R.; MATOS, D.S.; GERMANO, A.M.; JAMAL, C.M. Plantas medicinais utilizadas pela população atendida no "Programa de Saúde da Família", Governador Valadares, MG, Brasil. Revista Brasileira de Ciências Farmacêuticas, v. 44, n. 4, p. 629-636, 2008.

CAJAIBA, R.L.; CABRAL, J.A.; SANTOS, M. A Minimal Invasive Method to Forecast the Effects of Anthropogenic Disturbance on Tropical Cave Beetle Communities. Neotropical Entomology, v. 45, n. 2, p. 1-9, 2015. http://dx.doi.org/10.1007/s13744-015-0349-7.

CAJAIBA, R.L.; SILVA, W.B.; SOUSA, R.D.N; SOUSA, A.S. Levantamento etnobotânico de plantas medicinais comercializadas no município de Uruará, Pará, Brasil. Biotemas, v. 29, n. 1, p. 115-131, 2016. http://dx.doi.org/10.5007/21757925.2016v29n1p115.

CEOLIN, T.; HECK, R.M.; BARBIERI, R.L.; SCHWARTZ, E.; MUNIZ, R.M.; PILLON, C.N. Plantas medicinais: transmissão do conhecimento nas famílias de agricultores de base ecológica no Sul do RS. Revista da Escola de Enfermagem da USP, v. v. 45, n. 1., p. 47-54, 2011.

DOURADO, E.R.; DOCA, K.N.P.; ARAUJO, T.C.C. Comércio de produtos medicinais por "raizeiros" na cidade de Anápolis - GO. Revista Eletrônica de Farmácia, v. 2, n. 2, p. 67-69, 2005.

FERREIRA, J.M. Plantas de uso medicinal e ritualístico comercializadas em mercados e feiras no Norte do Espírito Santo, Brasil. Dissertação (Mestrado em Biodiversidade Tropical), Universidade Federal do Espírito Santo, 2014, 99p.

FREITAS, A.V.L.; COELHO, M.F.B.; AZEVEDO, R.A.B.; MAIA, S.S.S. Os raizeiros e a comercialização de plantas medicinais em São Miguel, Rio Grande do Norte, Brasil. Revista Brasileira de Biociências, v. 10, n. 2, p. 147-156, 2012. 
HOEFFEL, J.L.M.; GONÇALVES, N.M.; FADINI, A.A.B.; SEIXAS, S.R.C. Conhecimento tradicional e uso de plantas medicinais nas APAS's Cantareira/SP e Fernão Dias/MG. Revista VITAS, n. 1, p. 1-25, 2011.

MARQUES, J.G.W. Aspectos ecológicos na Etnoictiologia dos pescadores do complexo Estuarino Lagunar Mundaú Manguabá Alagoas. 1991. 280 p. Tese (Doutorado), Universidade Estadual de Campinas, Campinas. 1991.

OLIVEIRA, O.F.V.; GONDIM, M.J.C. Plantas medicinais utilizadas pela população de Caldas Novas, GO e o conhecimento popular sobre a faveira (Dimorphan dramollis Benth-Mimosoideae). Revista Brasileira de Agroecologia, v. 8, p. 156-169, 2013.

PRESS, I. Urban folk medicine: a functional overview. American Anthropologist, v. 80, p. 71-84, 1978.

ROCHA, F.A.G.; ARAÚJO, M.F.F.; COSTA, N.D.L.; SILVA, R.P.; QUEIROGA, P.V.D.M.; MARCIANO, L. A.; PONTES, E.D.M.; SOUZA, J.A.B. Características socioeconômicas dos comerciantes de plantas medicinais de Currais Novos/RN. Holos, v. 4, p. 87-100, 2013. 